5. Huicho L, Segura ER, Huayanay CA, Niño de Guzman J, Restrepo MC, Tam Y, et al. Child health and nutrition in Peru within an antipoverty political agenda: a Countdown to 2015 country case study. Lancet Global Health 2016: 4:e414-426

Correspondencia: Eduardo Casalino Rojo

Dirección: Los Damascos 359 La Molina-Lima

Correo electrónico: eduardo.casalino@gmail.com

\section{VALIDEZ DE ESTUDIOS PERUANOS SOBRE ESTRÉS Y BURNOUT}

\section{VALIDITY OF PERUVIAN STUDIES ON STRESS AND BURNOUT}

César Merino-Soto ${ }^{1,2, a}$, Gustavo Alexis Calderón-De la Cruz ${ }^{1, b}$

Sr. Editor. Desde el año 2014, el Instituto Nacional de Estadística e Informática (INEI) y la Superintendencia Nacional de Salud (SUSALUD) vienen desarrollando la Encuesta Nacional de Satisfacción de Usuarios en Salud (ENSUSALUD), cuyo contenido y datos están disponibles en: http://portales.susalud.gob.pe/web/portal/base-de-datos. Esta tiene como propósito explorar la percepción de los usuarios sobre el sistema de salud del Perú (1), así como algunas reacciones desadaptativas del personal de salud a su contexto laboral, específicamente, la evaluación del burnout (años 2014 y 2016) y el estrés (año 2015) en médicos y enfermeros. De estos se derivaron dos importantes estudios, uno de prevalencia del burnout (2), y otro sobre el estrés percibido y la intención de migrar al interior del Perú ${ }^{(3)}$. No obstante, en ningún caso se elaboró de forma previa un análisis psicométrico en la muestra de estudio.

Los instrumentos usados corresponden al Maslach Burnout Inventory-Human Survey (MBI-HSS), destinado para examinar el síndrome de burnout, y el Perceived Stress Scale (PSS), creado para evaluar la experiencia cognitiva del estrés. Ambas medidas son ampliamente conocidas en la investigación psicológica internacional, especialmente en el tópico de los factores psicosociales en el trabajo, y existen numerosas traducciones. En este punto, ¿sería necesario examinar las propiedades psicométricas de

\footnotetext{
Universidad Autónoma del Estado de Morelos. Morelos, México

Universidad de San Martín de Porres. Lima, Perú

Magister en Psicología; ${ }^{\text {b }}$ bachiller en Psicología

Recibido: 03/03/2018 Aprobado: 07/03/2018 En línea: 08/05/2018
}

Citar como: Merino-Soto C, Calderón-De la Cruz GA. Validez de estudios peruanos sobre estrés y burnout. Rev Peru Med Exp Salud Publica. 2018;35(2):3534. doi: 10.17843/rpmesp.2018.352.3521. estos instrumentos, aún si en otros contextos hay estudios sobre su validez?

La respuesta taxativa es sí, por varios motivos: primero, actualmente el estándar metodológico es la corroboración de las propiedades métricas de las medidas utilizadas, particularmente en contextos nuevos. Segundo, no hay ningún estudio peruano publicado que garantice que las propiedades métricas se mantengan satisfactorias. $Y$ tercero, la presunción de invariabilidad métrica (respecto a la validez y confiabilidad) no es garantizada por los estudios precedentes, y creerlo lleva a la situación de inducción de la validez, similar a la inducción de la confiabilidad ${ }^{(4)}$. En ambas situaciones, se mantienen las creencias erradas de que las propiedades específicas se conservan de un estudio a otro, y que esta información es suficiente respaldo para aplicarla a una nueva situación donde se aplica el mismo instrumento.

Dado que las propiedades métricas de ambos instrumentos son perfectamente corroborables con la metodología disponible y con los datos en mano, no parece justificable no corroborar sus propiedades psicométricas. Como corolario a todo lo anterior, el MBI-HSS frecuentemente ha sido cuestionado porque el número de dimensiones e ítems no se mantiene invariable entre los estudios ${ }^{(5,6)}$, un problema reconocido principalmente en contextos no anglosajones. Algunas consecuencias de este problema es que los puntajes son construidos sobre diferentes estructuras, la interpretación de los constructos cambia, el punto de corte (si es que existe alguno validado localmente) derivado de algún estudio anterior para identificar los casos de los no casos no tiene relevancia, y la prevalencia estimada es cuestionable y posiblemente inválida (al menos, hasta no demostrar lo contrario).

Considerando que la información con base científica requiere que se corroboren varios aspectos que contribuyen a su calidad (p. ej. validez y confiabilidad), no es suficiente confiar en los instrumentos, sino verificarlos empíricamente.

Contribución de autoría: CMS aportó en la concepción del estudio, redacción y aprobación final del manuscrito. GACC participo en la recolección de datos, redacción y aprobación final del manuscrito. Fuentes de Financiamiento: Universidad de San Martín de Porres.

Conflicto de Interés: No existe conflicto de interés entre los autores del manuscrito.

\section{REFERENCIAS BIBLIOGRÁFICAS}

1. Instituto Nacional de Estadística e Informática (INEI). Superintendencia Nacional de Aseguramiento en Salud (SUNASA). Encuesta nacional de satisfacción de usuarios del aseguramiento universal en salud. Lima: INEI/SUNASA; 2014

2. Maticorena-Quevedo J, Beas R, Anduaga-Beramendi A, Mayta-Trista P. (2014). Prevalencia del síndrome de burnout en médicos y enfermeras del Perú, ENSUSALUD 2014. Rev Peru 
Med Exp Salud Publica. 2016;33(2):241-7. doi: 10.17843/rpmesp.2016.332.2170.

3. Santiago-Ullero B, Valer-Villanueva S, Urrunaga-Pastor D, BenitezZapata VA. Estrés percibido e intención de migrar al interior del país en Médicos y Enfermeros que residen en Lima: un análisis exploratorio de la Encuesta Nacional de Satisfacción de Usuarios en Salud (ENSUSALUD), 2015. Rev Peru Med Exp Salud Pública. 2017;34(3):404-13. doi: 10.17843/rpmesp.2017.343.2822.

4. Sánchez-Meca J, Alacid-de-Pascual I, López-Pina JA, Cruz Sánchez-Jiménez J. Meta-análisis de generalización de la fiabilidad del inventario de obsesiones de Leyton versión para niños autoaplicada. Rev Esp Salud Publica. 2016;90:e1-e14.

5. Loera B, Converso D, Viotti S. Evaluating the psychometric properties of the Maslach Burnout Inventory-Human Services Suvery (MBI-HSS) among Italian nurses: how many factors must a researcher consider? PLoS One. 2014;9(12):e114987. doi: 10.1371/journal.pone.0114987.

6. Olivares-Faúndez VE, Mena-Miranda L, Jélvez-Wilke C, MacíaSepúlveda F. Validez factorial del Maslach Burnout Inventory Service (MBI-HSS) en profesionales chilenos. Univ Psychol. 2014;13(1):145-59. doi: 10.11144/Javeriana.UPSY13-1.vfmb.

Correspondencia: César Merino Soto

Dirección: Calle Vieja 322, interior 116, Condominio Las Bugambilias

2, CP: Cuernavaca, Morelos, México.

Teléfono: (52) 7773644210

Correoelectrónico:sikayax@yahoo.com.ar

\section{INCENTIVOS POR PUBLICACIÓN CIENTIIFICA EN UNIVERSIDADES PERUANAS QUE CUENTAN CON ESCUELAS DE MEDICINA, 2017}

\section{INCENTIVES FOR SCIENTIFIC PUBLICATION IN PERUVIAN UNIVERSITIES THAT HAVE MEDICAL SCHOOLS, 2017}

Wendy Nieto-Gutierrez ${ }^{1, a}$, José Ernesto Fernández-Chinguel ${ }^{1, a}$, Alvaro Taype-Rondan',b, Josmel Pacheco-Mendoza ${ }^{2, \mathrm{c}}$, Percy Mayta-Tristán ${ }^{3, d}$

Sr. Editor. Las universidades tienen entre sus principales funciones la generación de conocimiento, lo que se traduce, entre otras acciones, en publicaciones científicas en revistas

\footnotetext{
Facultad de Medicina Humana, Universidad de San Martín de Porres. Lima, Perú.

2 Centro de Investigación en Epidemiología Clínica y Medicina Basada en Evidencias. Instituto de Investigación, Facultad de Medicina Humana, Universidad de San Martín de Porres. Lima, Perú

Universidad San Ignacio de Loyola. Lima, Perú

4 Dirección General de Investigación, Desarrollo e Innovación, Universidad Científica del Sur. Lima, Perú

a Estudiante de Medicina; ${ }^{\mathrm{b}}$ médico epidemiólogo; ${ }^{\mathrm{c}}$ veterinario, magister en gestión de la información y el conocimiento; ${ }^{\mathrm{d}}$ médico salubrista Recibido: 21/12/2017 Aprobado: 17/01/2018 En línea:03/05/2018
}

Citar como: Nieto-Gutierrez W, Fernández-Chinguel JE, Taype-Rondan A, Pacheco-Mendoza J, Mayta-Tristán P. Incentivos por publicación científica en universidades peruanas que cuentan con escuelas de medicina, 2017. Rev Peru Med Exp Salud Publica. 2018;35(2):354-6. doi: 10.17843/rpmesp.2018.352.3327. indizadas. Es por ello que los rankings de universidades suelen valorar el número de publicaciones realizadas por autores afiliados a su universidad de procedencia.

Esto ha generado que las universidades implementen políticas para incentivar el desarrollo de la investigación, siendo una de estas la bonificación económica a los autores por las publicaciones que realizan en revistas científicas, esta estrategia ha demostrado elevar el número de publicaciones científicas en algunas instituciones ${ }^{(1)}$.

En Perú, luego de la reforma implementada con la Ley Universitaria $\mathrm{N}^{\circ} 30220$ del año 2014 (https://goo.gl/dui4kM), algunas universidades han implementado incentivos para que sus docentes, trabajadores y estudiantes incrementen la producción científica de sus instituciones. Sin embargo, no se cuentan con datos al respecto, por lo cual se realizó un estudio con el objetivo de describir los incentivos por publicación científica en universidades peruanas que cuentan con escuelas de medicina.

Se realizó un estudio observacional, descriptivo de corte transversal, cuyas unidades de análisis fueron las 57 escuelas de medicina del Perú, registradas hasta el 2016 (considerando las distintas filiales). Durante noviembre de 2017 se buscó información acerca de los incentivos económicos y no económicos que brindan estas escuelas o sus universidades por publicar en revistas científicas, durante el año 2017. Para ello, se consultaron las siguientes fuentes para cada escuela de medicina: 1) páginas web oficiales de las escuelas de medicina y las universidades, 2) entrevistas directas con los directores de las escuelas de medicina o llamadas telefónicas a los centros de administrativos de cada escuela, y 3) búsquedas en Google con los nombres de cada una de las universidades y los términos: «incentivos por publicación», «reconocimientos por publicación» y «pago económico por publicación».

De las 57 escuelas de medicina evaluadas el 2017, solo ocho brindaron algún incentivo económico; las cuales corresponden a seis universidades (puesto que dos escuelas fueron filiales). De estas, solo la Universidad Nacional San Antonio Abad del Cusco (UNSAAC) es de presupuesto público. Solo dos, además, otorgaban incentivos no económicos (diplomas). Las características de los financiamientos, según universidades, se muestran en la Tabla 1.

En cinco de las seis universidades, el monto del incentivo económico dependía de la base de datos donde estaba indizada la revista en la que se realizó la publicación. Sólo dos universidades, la Universidad Científica del Sur (UCSUR) y la Universidad Peruana de Ciencias Aplicadas (UPC) brindaron el incentivo económico diferenciado, acorde al cuartil de la revista (según Scopus o Web of Science) en la que se publicó el artículo.

Tres de las seis universidades comenzaron a brindar incentivos económicos desde el 2017, otra desde el 2016 